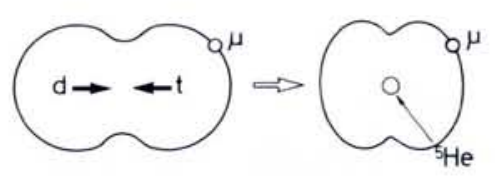

Fig. 5 - Fusion by chemical confinement within the dt $\mu$ molecule. At the instant of nuclear reaction, the molecular orbital of $d t \mu$ resembles the atomic orbital of $\left({ }^{5} \mathrm{He}\right) \mu$.

where $\lambda_{0}$ is the muon decay rate (inverse of its life-time) and $\lambda_{c}$ is the muon cycling rate (inverse average time between the consecutive fusions, determined mostly by the slowest process, namely the $d t \mu$ formation rate). This simplified expression reveals the role of the sticking fraction; it defines the ultimate limitation to the number of fusions per muon, as the cycling rate increases.

The experimental value for the effective sticking fraction, obtained independently of the fusion neutron disappearance rate is between 0.5 and $0.3 \%$ at the density 1.2 LHD. New experiments based on the direct detection of $\alpha \mu$ ions are under way in the form of joint ventures by RAL/LAMPF, and PSI/Gatchina. The current theoretical value of the initial sticking factor (obtained by means of three-body quantum chemical calculations) is $0.886 \%$. The main thrust of the research is to find a way to enhance the reactivation and lower the sticking fraction.

\section{PROSPECTS OF ENERGY PRODUCTION}

The principle of $\mu \mathrm{CF}$ is the same as that of a typical chemical catalysis:

$$
\begin{aligned}
& E_{\text {in }}+\mathrm{d}+\mathrm{t}+\mu \rightarrow \mathrm{dt} \mu \\
& \rightarrow \alpha+\mu+\mathrm{n}+E_{\text {out }}
\end{aligned}
$$

The remarkable reaction above occurs without any extra energy input, except

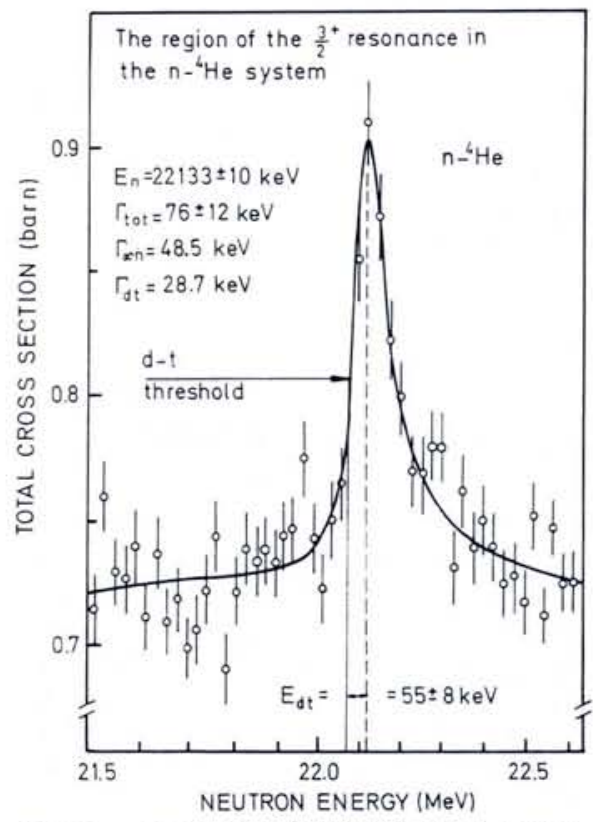

Fig. 6 -Cross-section for the $n-\alpha$ scattering in the vicinity of $3 / 2^{+}$resonance, with the energy above the $d$ - $t$ threshold and the total width estimated to be $E_{\text {res }}=55 \mathrm{keV}$ and $\Gamma_{\text {tot }}=76 \mathrm{keV}$, respectively.

the one needed for the production of muons $\left(E_{\text {in }}\right.$ is an energy of thermal motion, in the range of $\mathrm{meV}$ ). If $E_{\mu}$ denotes the energy needed for muon production, and $Y$ the neutron yield per muon, the condition for (scientific) energy breakeven is

$$
E_{\mu}<E_{\text {out }}=Y \times 17.6 \mathrm{MeV}
$$

It is clear that the key questions regarding possible energy production are: 1) can muons be produced cheaply, and 2) how many fusions can one muon promote during its short life time?

Recent experiments at LAMPF have demonstrated yields of 150 fusions/ muon, with energy liberation just short of $3 \mathrm{GeV} /$ muon, and with indications that yields of some 300 fusions/muon might be possible.

As to the cost of muons the current estimation is some $5 \mathrm{GeV}$ in beam power per muon, although the theoretical limit is below $2 \mathrm{GeV}$. Present muon factories are far from the optimal efficiency.

Along with the progress in theoretical and experimental research, different concepts of muon-catalysed fusion reactors are being developed. Pure fusion design is not possible today, but fusion-fission hybrids combined with a spallation breeder would require only

\title{
The Case of the Non-Stick Pan
}

It is becoming ever more probable that when all the conditions are carefully controlled, the electrolysis of $\mathrm{D}_{2} \mathrm{O}$ using a palladium cathode, will yield no energy gain and a negligible number of neutrons. So the table-top fusion reactor [1] is not for tomorrow. Both Cal. Tech. and MIT have failed to find any of the positive effects reported by Fleischmann and Pons [2] or even Jones et al. [3]. At Harwell, where they have had 30 cells running since $10 \mathrm{March}$ (some of which were set up in collaboration with Fleischmann) no heat gain or neutrons had been detected by the end of April. Again in EPFL Lausanne, nothing. So what do we conclude?

It is a little early to be $100 \%$ certain, but then absolute certainty in science is rather rare. However certain deficiencies have been noted in the reported Pons, Fleischmann experiments (which were run on a shoestring), notably the emergence of the cathode from the electrolyte and the relative primitive techniques used to measure the neutrons and monitor the background. Surface effects at the electrode are sufficient to explain the apparent calorimetric gain, tritium is a familiar contaminant and a variety some 100 fusions/muon, and could be operated with a net energy gain even if the cost of a muon stays as high as 5 GeV. Recent conceptual systems envision sidestepping the production of intermediate pion and muon beams. In these so called synergetic systems the $\mathrm{D} / \mathrm{T}$ mixture plays the role of both the target for muon production and the fuel for $\mu \mathrm{CF}$.

Finally, some safety considerations ought to be mentioned. Needless to say, thermal runaway of the muon catalysed fusion is not possible due to the selflimiting nature of the process at high temperatures. Since the muon catalysed fusion is a sort of resonant chemical reaction, the process is expected to proceed effectively only at the corresponding resonant temperature.

The process is still in an early stage of exploration. Only future research will show whether its efficiency is irrevocably given by Nature, or may be altered by scientific and technological progress.

\section{REFERENCES}

Further introductory reading can be found in:

Rafelski J. and Jones S.E., Sci. Amer. 257 (1987) 1, 84

Ponomarev L.I. and Fiorentini G., Muon Catalysed Fusion 1 (1987) 3.

Jones S.E., Nature 321 (1986) 127.

Bracci L. and Fiorentini G., Physics Reports 86 (1982) 171.

Gershtein et al., Sov. Phys. JETP 53 (1981) 872.

Petrov Y.V., Nature 285 (1980) 466. of mechanisms have been invoked to account for the seemingly excess neutrons, ranging from radon emission in the laboratory to high electric fields generated in the lattice - a sort of micro piezoelectric effect [4].

The moral to the story which should be pointed out to the lay man and the politicians, is not that individual scientists may be fallible, which we know, but that the esta blishment can be relied on to get it right in the end. Moreover the establishment is not so arrogant that it declines to examine something new when it comes along.

\section{REFERENCES}

[1] Shaw E.N., Electro-chemical Induced Fusion, Europhys. News 20 (1989) 45.

[2] Fleischmann M. and Pons S., 'Electrochemically Induced Nuclear Fusion of Deuterium', J. Electroanal. Chem. 261 (1989) 301.

[3] Jones S.E. et al., 'Observation of Cold Nuclear Fusion in Condensed Matter' Nature 338 (1989) 737.

[4] Klyuev V.A. et al., 'High-energy Proces ses Accompanying the Fracture of Solids', Pis'ma Tekh. Fiz. 12 (1986) 1333. 